\title{
Chemical transport associated with discharge of contaminated fine particles to a steady open-channel flow
}

\author{
Chiu-On Ng \\ Department of Mechanical Engineering, The University of Hong Kong, Pokfulam Road, Hong Kong
}

(Received 4 December 1998; accepted 29 September 1999)

\begin{abstract}
In this paper, an analytical study on the advective-dispersive transport of a chemical contaminant resulting from the discharge of contaminated fine solid particles into a two-dimensional, steady and uniform turbulent open-channel flow is presented. Because of sorptive exchange, the transport of the chemical cloud is affected by that of the suspended particulates. Such a relationship has so far not been explicitly established by intuitive arguments. The effective transport equations are formally derived by an extended method of homogenization. It is found that over a long time scale the fall velocity will delay the sediment advection, and the advection velocity and dispersion coefficient for the chemical transport will change with space and time according to the local sediment concentration. Numerical results confirm that the centers of mass of the sediment and dissolved phase clouds are not advancing at the same speed, and the dispersion of the chemical is enhanced by the local retardation factor. (C) 2000 American Institute of Physics. [S1070-6631(00)01501-4]
\end{abstract}

\section{INTRODUCTION}

Sediments are now recognized as an important carrier of hazardous substances such as heavy metals, PCBs (polychlorinated biphenyls), PAHs (polycyclic aromatic hydrocarbons), and pesticides in aquatic systems. Chemicals carried by suspended load typically will change in concentration or composition in response to the local aquatic environment via phase change, reaction, degradation, uptake by organisms, and so on. Despite the sizable literature on sediment transport and water quality control, the understanding of the influence of sediment movement on the physico-chemical fate of a contaminant is rather limited.

In this work we focus on the mass transport of a chemical resulting from the dumping of contaminated wastes into a two-dimensional open channel. The dumped matters are essentially fine solid particulates that remain in suspension throughout their transport along the channel. Initially the wastes are contaminated; a chemical pollutant which is conservative, nonvolatile, and nonreactive is carried by the solid particles. Upon discharge to the water, a fraction of the chemical dissolves as an aqueous phase, while the rest remains sorbed onto the suspended particulates. If the chemical sorptivity and the particle concentration are large enough, the fraction of chemical in sorbed form can be comparable to that in dissolved form. If so, fluxes of both sorbed and dissolved forms must be accounted for in order to determine the transport rate of the chemical. While the two phases are carried by different agents which are subject to different transport mechanisms, there is a continuous local phase exchange (sorption and desorption) between the aqueous and the sorbed species. The phase exchange coupled with a possible difference in velocity of the two agents would render the transport of the dissolved phase to be affected by that of the sediment. The mechanics of such a relationship has thus far not been studied. The objective of the present study is to develop a theory for the transport of a chemical which because of sorptive exchange is associated with the transport of suspended particulates.

Both advection and dispersion of the dissolved phase are expected to be affected by those of the particulates. In transport modeling it is always a nontrivial matter to determine the dispersion coefficients, which can vary over a wide range depending on the flow kinematics and the channel configuration. Dispersion is the combined effects of longitudinal advection and lateral diffusion, and its coefficient can in principle be found from the cross-sectional covariance of velocity and concentration. Based on the pioneering work of Taylor, ${ }^{1,2}$ Elder $^{3}$ derived the dispersion coefficients for the spreading of a conservative substance in a two-dimensional, steady and uniform turbulent open-channel flow. Since then, many have contributed to establishing empirical methods for estimation of dispersion coefficients in natural streams (e.g., see the list compiled by Schnoor ${ }^{4}$ ). All these works however focus only on the dispersion of a single matter; the case of dispersion of a substance under the influence of phase exchange with another form appears not to have received much attention. In fact, it is not clear how conventional techniques such as the theory of diffusion by continuous movements used by Taylor ${ }^{2}$ and Elder $^{3}$ and the moment method used by Aris ${ }^{5}$ can readily be extended for the present problem.

To achieve our goal, we shall derive effective equations by following the asymptotic method of homogenization, which was introduced by Sanchez-Palencia ${ }^{6}$ and epitomized by, among others, Bensoussan et al. ${ }^{7}$ The homogenization method is essentially an averaging method based on the mathematical techniques of multiple scales, and is particularly useful for materials with a periodic microstructure. It is capable of yielding phenomenological equations on the basis of micro-mechanics, in a general manner without any closure hypotheses. Application of this method to a variety of mechanics problems has recently been reviewed by Mei et al. ${ }^{8}$ 
In particular, the method has been extensively used to study mass and thermal dispersion in porous media, ${ }^{9-13}$ in layered porous media, ${ }^{14}$ and in a wave boundary layer. ${ }^{15,16}$ It is remarkable that in the deduced effective transport equations, the advection velocity and dispersion coefficients are expressible in terms of some cell functions which for onedimensional microstructures can usually be solved analytically.

To prepare grounds for application of the homogenization method, we shall further define our problem in Sec. II and clearly state the assumptions. Orders of magnitude of the physical quantities are estimated in terms of a small parameter given by the ratio of the eddy diffusivity to the longitudinal dispersion coefficient. Specific conditions include: a much longer longitudinal length scale than the flow depth, a much faster transport by advection than by vertical eddy diffusion, a much smaller settling velocity of particulates than the mean stream velocity, a much longer time scale for longitudinal dispersion than advection, and comparable fractions of chemical in sorbed and dissolved forms. In terms of the small ordering parameter, perturbation expansions followed by depth averaging of the sediment and the chemical transport equations are carried out in Secs. III and IV, respectively. It will be seen at the leading order that both substances are advected at the mean stream velocity. More interesting and original results show up at the next order, whose effects become significant over a long time of transport. For the sediment transport, we recover the longitudinal dispersion coefficient as obtained heuristically by Elder. ${ }^{3}$ It is also found that, based on a parabolic eddy diffusivity distribution, the sediment advection is actually delayed by a speed amounting to approximately ten times the fall velocity. For the chemical transport, the retardation factor, the advection velocity, and the dispersion coefficient are all functions of the local sediment concentration or its spatial gradient. Such functional dependence means the influence of the sediment transport on the chemical transport. An illustrative example is presented in Sec. V where a pulse input of waste disposal is considered. Discussion is focused on the effects of the fall velocity and the chemical solid-water distribution ratio on the spreading of the dissolved chemical cloud.

\section{SCALINGS AND BASIC TRANSPORT EQUATIONS}

A two-dimensional, steady and uniform turbulent channel flow is considered. The concentrations of the dissolved matter and the suspended particles are so small that the flow is not materially altered by their presence. The turbulence is strong enough to place the particles in suspension with a negligible net rate of deposition. On the other hand, the channel bottom is well consolidated so that no scouring takes place either. We also assume a local equilibrium partitioning between the dissolved and particulate chemical. This assumption is valid as long as the time to equilibration is much shorter than the transport time scales, which is realizable when the mass transfer is not rate limited by diffusive processes. This condition is met when particles are small enough and the diffusivity is large enough. We further assume that the channel bottom is free of organic matters so that no sorption takes place there. Effects of other chemical processes like biodegradation, volatilization, and photolysis are also ignored.

An $(x, z)$ coordinate system is defined with the $x$ axis along and the $z$ axis normal to the bottom of the channel. A small slope is considered so that the $z$ direction is virtually pointing vertically upward. The normal depth is $h$, and the time-smoothed longitudinal velocity is $u(z)$. When suspended, the solid particles behave like fluid particles except that they tend to settle with a fall velocity, whose timeaveraged value $w_{f}$ is assumed to be a constant in this study. The longitudinal and the vertical eddy diffusivities, denoted by $E_{x}(z)$ and $E_{z}(z)$, respectively, are assumed to be the same for both fluid mass and sediment particles. The agreement has been supported by experiments for fine particles with a nominal diameter less than $0.1 \mathrm{~mm}$ (e.g., Sayre ${ }^{17}$ ), or with a ratio of fall velocity to shear velocity less than 0.2 (e.g., Rijn ${ }^{18}$ ). Also for small particles, the advection velocity is essentially equal to the mean velocity of the local fluid. ${ }^{15}$ With these assumptions, the transport equation for the suspended sediment can be written as

$$
\frac{\partial \zeta}{\partial t}+u \frac{\partial \zeta}{\partial x}-w_{f} \frac{\partial \zeta}{\partial z}=\frac{\partial}{\partial x}\left(E_{x} \frac{\partial \zeta}{\partial x}\right)+\frac{\partial}{\partial z}\left(E_{z} \frac{\partial \zeta}{\partial z}\right),
$$

where $t$ is the time and $\zeta(x, z, t)$ is the sediment concentration (mass of suspended particles per bulk volume). No sediment loss occurs on the bottom and the top of the channel, so the deposition and entrainment rates must be balanced there:

$$
w_{f} \zeta+E_{z} \frac{\partial \zeta}{\partial z}=0 \quad \text { at } z=0, h .
$$

As driven by thermodynamics, the chemical is always partitioned between the aqueous and the solid phases. Under local equilibrium partitioning, a linear sorption isotherm may be used to relate the aqueous concentration $C(x, z, t)$ (mass of dissolved species per bulk volume) and the sorbate concentration $C_{s}(x, z, t)$ (mass of sorbed species per mass of sediment):

$$
K_{d}=C_{s} / C
$$

where $K_{d}$ is the sorption partition coefficient which depends on the chemical and the sediment properties. Hence, the total chemical mass concentration $C_{\text {tot }}(x, z, t)$ is

$$
C_{\text {tot }}=C+C_{s} \zeta=C\left(1+K_{d} \zeta\right)
$$

which is governed by the transport equation

$\frac{\partial C_{\mathrm{tot}}}{\partial t}+u \frac{\partial C_{\mathrm{tot}}}{\partial x}-w_{f} \frac{\partial C_{s} \zeta}{\partial z}=\frac{\partial}{\partial x}\left(E_{x} \frac{\partial C_{\mathrm{tot}}}{\partial x}\right)+\frac{\partial}{\partial z}\left(E_{z} \frac{\partial C_{\mathrm{tot}}}{\partial z}\right)$,

and the zero-flux boundary conditions

$$
w_{f} C_{s} \zeta+E_{z} \frac{\partial C_{\text {tot }}}{\partial z}=0 \quad \text { at } \quad z=0, h .
$$

While the sediment transport is independent of the chemical transport, the chemical transport is, however, affected by the sediment transport. Also by (2), (6) can be simplified to 


$$
E_{z}\left(\frac{\partial C}{\partial z}+\zeta \frac{\partial C_{s}}{\partial z}\right)=0 \quad \text { at } z=0, h .
$$

To facilitate subsequent perturbation analysis, let us estimate and relate the scalings of various quantities.

(1) Longitudinal dispersion coefficient versus eddy diffusivity. Although in the above-mentioned equations the longitudinal diffusion is controlled by the eddy diffusivity $E_{x}$, it is anticipated that, after a long enough time of transport, the effective longitudinal diffusion will be dominated by the dispersion $D$ resulting from the velocity variation associated with turbulent diffusion in the $z$ direction. Elder ${ }^{3}$ has found that for a steady turbulent channel flow

$$
\bar{E}_{x}=0.07 u_{*} h, \quad D=5.86 u_{*} h,
$$

where the overhead bar denotes the depth average, and $u_{*}$ is the shear velocity that can be calculated from the channel slope $\theta$ :

$$
u_{*}=\sqrt{g h \sin \theta} .
$$

Clearly the dispersion coefficient $D$ is much larger than the turbulent diffusivity $E$, so we may put

$$
E / D=\varepsilon \ll 1 .
$$

In the following, we shall normalize the longitudinal diffusion with respect to $D$, and the vertical diffusion with respect to $E$. The small parameter $\varepsilon$ will be used as the perturbation parameter.

(2) The longitudinal and vertical length scales. As in most dispersion studies, we shall focus on the spread of matters at a large time after initial discharge. The longitudinal scale $L$ for the spreads of the chemical and the particles will then be much larger than the flow depth $h$. In order that the longitudinal dispersion is effectively two orders smaller than the vertical diffusion, we assume that

$$
h / L=O\left(\varepsilon^{3 / 2}\right) .
$$

(3) Ratio of advection to vertical diffusion. This ratio is a Péclet number, and on using (8) can be estimated as follows:

$$
\mathrm{Pe}_{z}=\frac{\bar{u} h}{E}=O\left(\frac{\bar{u}}{0.07 u_{*}}\right),
$$

where $\bar{u}$ is the depth-averaged velocity. Since typically $\bar{u}$ $\geqslant u_{*}$, the above-mentioned number is much larger than unity. In order that the longitudinal advection is effectively one order smaller than the vertical diffusion, we assume that

$$
\mathrm{Pe}_{z}=O\left(\varepsilon^{-1 / 2}\right) \text {. }
$$

(4) Ratio of fall velocity to flow velocity. The fall velocity depends on the size and shape of the particles. ${ }^{19}$ For fine sands and coarse silts of $0.03-0.1 \mathrm{~mm}$ nominal diameter and unity shape factor, the fall velocity is on the order of 1-10 $\mathrm{mm} / \mathrm{s}$. This is much smaller than the stream flow velocity, typically on the order of $1-10 \mathrm{~m} / \mathrm{s}$. Hence, we may put

$$
W_{f} / \bar{u}=O\left(\varepsilon^{3 / 2}\right) .
$$

(5) Time scales. Two time scales will be pertinent corresponding to the two transport processes. For a longitudinal stretch $L$, it takes $T_{1}=L / \bar{u}$ to travel by advection, while a much longer time $T_{2}=L^{2} / D$ is needed for spreading by dispersion, as shown below:

$$
T_{2}=\frac{L^{2}}{D}=\frac{L}{h} \frac{E}{D} \frac{\bar{u} h}{E} \frac{L}{\bar{u}}=O\left(\varepsilon^{-1}\right) T_{1},
$$

where (10), (11), and (13) have been used.

(6) Bulk solid-water distribution ratio of chemical. In this study, we assume that the fractions of chemical in water and on solid are comparable so that both phases are important in the transport. The bulk solid-water distribution ratio, defined below, must therefore be of order unity:

$$
K_{d} \bar{\zeta}=O(1),
$$

where $\bar{\zeta}$ is a scale of the sediment concentration. For particles brought in by human dumping, the sediment concentration may vary over a wide range. If the flow is not to be affected by the presence of the particles, $\bar{\zeta}$ can be as much as $O(1) \mathrm{kg} / \mathrm{m}^{3}$. This is indeed the order of natural sediment concentration in the Yellow River mouth. ${ }^{16}$ With such a maximum order for $\bar{\zeta}$, condition (16) is met when $K_{d}$ $\geqslant O\left(10^{3}\right) \mathrm{L} / \mathrm{kg}$. Nonreactive and neutral organic chemicals typically show greater sorption coefficients for sediments with large amounts of organic matter. ${ }^{20}$ For example, Means et $a l .{ }^{21}$ have found the sorption partition coefficient for a hydrophobic compound, pyrene, to be on the order 1200 $\mathrm{L} / \mathrm{kg}$ when the organic carbon content of the solid is more than $2 \%$ by weight. Also, Wu and Gschwend ${ }^{22}$ found that the sorption partition coefficient for a number of river bed sediments can be as high as $4700 \mathrm{~L} / \mathrm{kg}$ when the sorbates are chlorobenzenes. Heavy metals and PCBs also have very high partition coefficients on the order of $10^{4}-10^{6} \mathrm{~L} / \mathrm{kg}$. In short, (16) can be realized when both the particle concentration and the sorption partition coefficient are sufficiently large.

Based on the above scalings, we introduce the following normalized quantities (distinguished by a caret):

$$
\begin{aligned}
& x=L \hat{x}, \quad z=h \hat{z}, \quad t=(L / \bar{u}) \hat{t}, \quad E_{x}=D \hat{E}_{x}, \\
& E_{z}=E \hat{E}_{z} .
\end{aligned}
$$

In terms of these normalized quantities, the sediment transport equations (1) and (2) can be written as

$$
\begin{aligned}
&\left(\frac{\bar{u} h}{E}\right) {\left[\left(\frac{h}{L}\right) \frac{\partial \zeta}{\partial \hat{t}}+\left(\frac{h}{L}\right) \hat{u} \frac{\partial \zeta}{\partial \hat{x}}-\left(\frac{w_{f}}{\bar{u}}\right) \frac{\partial \zeta}{\partial \hat{z}}\right] } \\
&=\left(\frac{D}{E}\right)\left(\frac{h}{L}\right)^{2} \frac{\partial}{\partial \hat{x}}\left(\hat{E}_{x} \frac{\partial \zeta}{\partial \hat{x}}\right)+\frac{\partial}{\partial \hat{z}}\left(\hat{E}_{z} \frac{\partial \zeta}{\partial \hat{z}}\right), \\
&\left(\frac{w_{f}}{\bar{u}}\right)\left(\frac{\bar{u} h}{E}\right) \zeta+\hat{E}_{z} \frac{\partial \zeta}{\partial \hat{z}}=0 \quad \text { at } \hat{z}=0,1 .
\end{aligned}
$$

Obviously, the terms on the left-hand side of (18) are of order $\varepsilon$, while the first term on the right-hand side is of order $\varepsilon^{2}$. Also the first term on the left-hand side of (19) is of order 
$\varepsilon$. Similar orders of magnitude can be obtained after normalization for the chemical concentration equation and boundary conditions (5) and (6).

Let us now revert to physical equations, but insert $\varepsilon$ 's for order identification. The sediment transport equation is

$$
\varepsilon \frac{\partial \zeta}{\partial t}+\varepsilon u \frac{\partial \zeta}{\partial x}-\varepsilon w_{f} \frac{\partial \zeta}{\partial z}=\varepsilon^{2} \frac{\partial}{\partial x}\left(E_{x} \frac{\partial \zeta}{\partial x}\right)+\frac{\partial}{\partial z}\left(E_{z} \frac{\partial \zeta}{\partial z}\right),
$$

with the boundary conditions

$$
\varepsilon w_{f} \zeta+E_{z} \frac{\partial \zeta}{\partial z}=0 \quad \text { at } z=0, h .
$$

The chemical transport equation is

$$
\begin{aligned}
& \varepsilon \frac{\partial C_{\text {tot }}}{\partial t}+\varepsilon u \frac{\partial C_{\text {tot }}}{\partial x}-\varepsilon w_{f} \frac{\partial C_{s} \zeta}{\partial z} \\
& =\varepsilon^{2} \frac{\partial}{\partial x}\left(E_{x} \frac{\partial C_{\text {tot }}}{\partial x}\right)+\frac{\partial}{\partial z}\left(E_{z} \frac{\partial C_{\text {tot }}}{\partial z}\right),
\end{aligned}
$$

with the boundary conditions

$$
\varepsilon w_{f} C_{s} \zeta+E_{z} \frac{\partial C_{\text {tot }}}{\partial z}=0 \text { at } z=0, h .
$$

The chemical transport equation and boundary conditions are to be expressed in terms of the aqueous concentration $C$ upon replacing $C_{\text {tot }}$ and $C_{s}$ by (4) and (3), respectively. Perturbation equations are obtained on substituting the following multiple-scale expansions into equations (20)-(23):

$$
\begin{aligned}
& \zeta \rightarrow \zeta_{0}+\varepsilon \zeta_{1}+\varepsilon^{2} \zeta_{2}+O\left(\varepsilon^{3}\right), \\
& C \rightarrow C_{0}+\varepsilon C_{1}+\varepsilon^{2} C_{2}+O\left(\varepsilon^{3}\right), \\
& \partial / \partial t \rightarrow \partial / \partial t_{1}+\varepsilon \partial / \partial t_{2} .
\end{aligned}
$$

\section{SEDIMENT TRANSPORT}

At $O(1)$, the problem is homogeneous:

$$
\frac{\partial}{\partial z}\left(E_{z} \frac{\partial \zeta_{0}}{\partial z}\right)=0, \quad 0<z<h,
$$

with the boundary conditions

$$
E_{z} \frac{\partial \zeta_{0}}{\partial z}=0, \quad z=0, h
$$

Clearly, $\zeta_{0}$ is independent of $z$, or

$$
\zeta_{0}=\zeta_{0}(x, t) .
$$

This is consistent with the well-known observation that particles with a small fall velocity will have a rather uniform equilibrium concentration profile. $^{23}$

At $O(\varepsilon), \zeta_{1}$ represents the component that varies with $z$, essentially due to the velocity variation and the fall velocity in this direction. The perturbation equation is

$$
\frac{\partial \zeta_{0}}{\partial t_{1}}+u \frac{\partial \zeta_{0}}{\partial x}-w_{f} \frac{\partial \zeta_{0}}{\partial z}=\frac{\partial}{\partial z}\left(E_{z} \frac{\partial \zeta_{1}}{\partial z}\right)
$$

and the boundary conditions are

$$
w_{f} \zeta_{0}+E_{z} \frac{\partial \zeta_{1}}{\partial z}=0 \quad \text { at } z=0, h
$$

On taking average over the depth, and using the boundary conditions, (30) yields the leading order equation for the sediment transport

$$
\frac{\partial \zeta_{0}}{\partial t_{1}}+\bar{u} \frac{\partial \zeta_{0}}{\partial x}=0
$$

where $\bar{u}=h^{-1} \int_{0}^{h} u d z$. As expected, only advection appears in Eq. (32). On subtracting (32) from (30), we get

$$
(u-\bar{u}) \frac{\partial \zeta_{0}}{\partial x}=\frac{\partial}{\partial z}\left(E_{z} \frac{\partial \zeta_{1}}{\partial z}\right)
$$

By linearity, (33) and (31) suggest the following form for $\zeta_{1}$ :

$$
\zeta_{1}=N \frac{\partial \zeta_{0}}{\partial x}+M \zeta_{0}
$$

where $N(z)$ and $M(z)$ are governed by the following boundary value problems:

$$
\begin{aligned}
& \frac{d}{d z}\left(E_{z} \frac{d N}{d z}\right)=u-\bar{u}, \quad 0<z<h, \\
& \frac{d N}{d z}=0, \quad z=0, h,
\end{aligned}
$$

and

$$
\begin{aligned}
& \frac{d}{d z}\left(E_{z} \frac{d M}{d z}\right)=0, \quad 0<z<h, \\
& E_{z} \frac{d M}{d z}=-w_{f}, \quad z=0, h .
\end{aligned}
$$

Solutions to the above problems Eqs. (35)-(38) are given in the Appendix.

At $O\left(\varepsilon^{2}\right)$, the perturbation equation is

$\frac{\partial \zeta_{0}}{\partial t_{2}}+\frac{\partial \zeta_{1}}{\partial t_{1}}+u \frac{\partial \zeta_{1}}{\partial x}-w_{f} \frac{\partial \zeta_{1}}{\partial z}=\frac{\partial}{\partial x}\left(E_{x} \frac{\partial \zeta_{0}}{\partial x}\right)+\frac{\partial}{\partial z}\left(E_{z} \frac{\partial \zeta_{2}}{\partial z}\right)$,

and the boundary conditions are

$$
w_{f} \zeta_{1}+E_{z} \frac{\partial \zeta_{2}}{\partial z}=0 \quad \text { at } \quad z=0, h .
$$

Using the boundary conditions, the depth-average of (39) gives

$$
\frac{\partial \zeta_{0}}{\partial t_{2}}+\frac{\partial \bar{\zeta}_{1}}{\partial t_{1}}+\overline{u \frac{\partial \zeta_{1}}{\partial x}}=\frac{\partial}{\partial x}\left(\bar{E}_{x} \frac{\partial \zeta_{0}}{\partial x}\right) .
$$

Further replacing $\zeta_{1}$ by (34), we obtain the second-order sediment transport equation, in which dispersion first appears:

$$
\frac{\partial \zeta_{0}}{\partial t_{2}}+u^{\prime} \frac{\partial \zeta_{0}}{\partial x}=\left(\bar{E}_{x}+D\right) \frac{\partial^{2} \zeta_{0}}{\partial x^{2}},
$$

where $u^{\prime}$ is the second-order velocity

$$
u^{\prime}=\overline{M u}-\bar{M} \bar{u},
$$


and $D$ is the dispersion coefficient

$$
D=\bar{N} \bar{u}-\overline{N u} \text {. }
$$

Finally, (32) and (42) are combined to give the depthaveraged sediment transport equation with an accuracy up to $O(\varepsilon)$ :

$$
\frac{\partial \zeta_{0}}{\partial t}+\frac{\partial J}{\partial x}=0,
$$

where $J$ is the total sediment flux due to advection and dispersion:

$$
J=\left(\bar{u}+\varepsilon u^{\prime}\right) \zeta_{0}-\varepsilon\left(\bar{E}_{x}+D\right) \frac{\partial \zeta_{0}}{\partial x} .
$$

As noted earlier, the small parameter $\varepsilon$ is inserted only for order identification, and should be omitted in computations.

Details of deriving expressions for $u^{\prime}$ and $D$ are presented in the Appendix. Only the key results are cited in the following. For any velocity profile, we may write

$$
\begin{aligned}
& u^{\prime}=-\frac{w_{f}}{h} \int_{0}^{h} \frac{d z}{E_{z}} \int_{z}^{h}(u-u) d z^{\prime}, \\
& D=\frac{1}{h} \int_{0}^{h} \frac{d z}{E_{z}}\left[\int_{z}^{h}(u-\bar{u}) d z^{\prime}\right]^{2} .
\end{aligned}
$$

On using the well-known logarithmic profile for turbulent stream flows, and, by Reynolds analogy, the parabolic eddy diffusivity distribution as follows:

$$
\begin{aligned}
& u(z)=\bar{u}+\frac{u_{*}}{\kappa}\left(\ln \frac{z}{h}+1\right), \\
& E_{z}(z)=u_{*} \kappa h \frac{z}{h}\left(1-\frac{z}{h}\right),
\end{aligned}
$$

where $\kappa=0.41$ is von Karman's constant, we may obtain explicit expressions:

$$
\begin{aligned}
& u^{\prime}=-\frac{\pi^{2}}{6 \kappa^{2}} w_{f}=-9.8 w_{f}, \\
& D=2 \sum_{n=2}^{\infty} n^{-3} \kappa^{-3} u_{*} h=5.86 u_{*} h .
\end{aligned}
$$

Also, assuming that the depth-averaged horizontal and vertical eddy diffusivities are equal, we may obtain

$$
\bar{E}_{x}=\bar{E}_{Z}=0.068 u_{*} h .
$$

The above-mentioned dispersion coefficient and mean eddy diffusivity are exactly those obtained by Elder. ${ }^{3}$ Our deductions have been more systematic, with the regime of validity clearly prescribed. Many limiting assumptions required in Elder's approach have also been avoided. The second-order velocity, an original finding, is approximately ten times the fall velocity. Since it is assumed that $w_{f}=O\left(\varepsilon^{3 / 2}\right) \bar{u}, u^{\prime}$ $\cong-10 w_{f}$ can be of order $\varepsilon \bar{u}$. It is remarkable that the sediment particles are actually transported at a speed that is smaller than the mean stream velocity by a value equal to ten times the fall velocity. The effect of this small reduction in advection speed will become appreciable after a long time of travel. The retardation of the sediment relative to the mean stream is due to the fact that, because the sediment particles are heavy, their concentration distribution is skewed toward the channel bottom, where the fluid velocity is the lowest.

\section{CHEMICAL TRANSPORT}

Let us now focus on the chemical transport, whose leading order equation and boundary conditions are

$$
\begin{aligned}
& \frac{\partial}{\partial z}\left[E_{z} R \frac{\partial C_{0}}{\partial z}\right]=0, \\
& E_{z} R \frac{\partial C_{0}}{\partial z}=0 \quad \text { at } z=0, h,
\end{aligned}
$$

where $R(x, t) \geqslant 1$ is the retardation factor

$$
R=1+K_{d} \zeta_{0}
$$

It is clear that $C_{0}$ is also independent of $z$, or

$$
C_{0}=C_{0}(x, t) \text {. }
$$

At $O(\varepsilon)$, the equation after simplification using (30) can be written as

$$
\frac{\partial C_{0}}{\partial t_{1}}+u \frac{\partial C_{0}}{\partial x}=\frac{\partial}{\partial z}\left(E_{z} \frac{\partial C_{1}}{\partial z}\right) .
$$

The boundary conditions are

$$
E_{z} \frac{\partial C_{1}}{\partial z}=0 \quad \text { at } z=0, h
$$

Upon taking depth average of (58), and using (59), we get the leading order chemical transport equation

$$
\frac{\partial C_{0}}{\partial t_{1}}+\bar{u} \frac{\partial C_{0}}{\partial x}=0,
$$

which resembles (32). Not surprisingly, the sediment and the chemical are transported at the leading order by advection of the same magnitude. There are, however, disparities in the advection and dispersion of the two matters at the next order, as will be seen in the following.

If we subtract (60) from (58), we obtain

$$
(u-\bar{u}) \frac{\partial C_{0}}{\partial x}=\frac{\partial}{\partial z}\left(E_{z} \frac{\partial C_{1}}{\partial z}\right) .
$$

Equation (61) for $C_{1}$ is like (33), the one for $\zeta_{1}$. We may immediately write

$$
C_{1}=N \frac{\partial C_{0}}{\partial x}
$$

where $N(z)$ is the function governed by (35) and (36).

At $O\left(\varepsilon^{2}\right),(22)$ yields, after averaging over the depth and using the boundary conditions (23) and simplifying by (41), 


$$
\begin{gathered}
R\left(\frac{\partial C_{0}}{\partial t_{2}}+\frac{\partial \bar{C}_{1}}{\partial t_{1}}\right)+K_{d}\left(\bar{C}_{1} \frac{\partial \zeta_{0}}{\partial t_{1}}+\bar{\zeta}_{1} \frac{\partial C_{0}}{\partial t_{1}}\right)+R \frac{\partial \overline{u C_{1}}}{\partial x} \\
+K_{d}\left(\overline{u C_{1}} \frac{\partial \zeta_{0}}{\partial x}+\overline{u \zeta_{1}} \frac{\partial C_{0}}{\partial x}\right) \\
=\frac{\partial}{\partial x}\left(R \bar{E}_{x} \frac{\partial C_{0}}{\partial x}\right)+K_{d} \bar{E}_{x} \frac{\partial \zeta_{0}}{\partial x} \frac{\partial C_{0}}{\partial x} .
\end{gathered}
$$

Further replacing $\zeta_{1}$ by (34), $\partial \zeta_{0} / \partial t_{1}$ by (32), $C_{1}$ by (62), and $\partial C_{0} / \partial t_{1}$ by (60), and after some manipulations, Eq. (63) gives the second-order chemical transport equation:

$$
\begin{gathered}
R \frac{\partial C_{0}}{\partial t_{2}}+K_{d}\left[u^{\prime} \zeta_{0}-\left(\bar{E}_{x}+D\right) \frac{\partial \zeta_{0}}{\partial x}\right] \frac{\partial C_{0}}{\partial x} \\
=\frac{\partial}{\partial x}\left[R\left(\bar{E}_{x}+D\right) \frac{\partial C_{0}}{\partial x}\right],
\end{gathered}
$$

where $u^{\prime}$ and $D$, defined earlier, respectively, in (43) and (44), are the second-order velocity and dispersion coefficient for the sediment transport. Explicit expressions for $u^{\prime}, D$ and $\bar{E}_{x}$ have also been obtained, respectively, in (51), (52), and (53) for a turbulent channel flow.

Combining (60) and (64), we finally get the resultant depth-averaged chemical transport equation with an accuracy up to $O(\varepsilon)$ :

$$
R \frac{\partial C_{0}}{\partial t}+\left(\bar{u}+K_{d} J\right) \frac{\partial C_{0}}{\partial x}=\varepsilon \frac{\partial}{\partial x}\left[R\left(\bar{E}_{x}+D\right) \frac{\partial C_{0}}{\partial x}\right],
$$

where $J$ is the flux of sediment, as defined in (46). Again the small parameter $\varepsilon$ serves only to indicate the order of the associated term and should be omitted in computations. On comparing Eq. (65) with its counterpart (45) for the sediment transport, it is remarkable that, because of sorptive exchange, the chemical transport is indeed associated with the sediment transport. First, the retardation factor $R=1+K_{d} \zeta_{0}$ depends on the local sediment concentration. Second, the advection velocity of the chemical has a component contributed by the net flux of the sediment. On dividing Eq. (65) by $R$ and expanding the dispersion term, the equation can also be written as follows:

$$
\begin{aligned}
\frac{\partial C_{0}}{\partial t} & +\left\{\bar{u}+\varepsilon \frac{K_{d}}{R}\left[u^{\prime} \zeta_{0}-2\left(\bar{E}_{x}+D\right) \frac{\partial \zeta_{0}}{\partial x}\right]\right\} \frac{\partial C_{0}}{\partial x} \\
& =\varepsilon\left(\bar{E}_{x}+D\right) \frac{\partial^{2} C_{0}}{\partial x^{2}} .
\end{aligned}
$$

Now, the effects of sorptive exchange on the chemical transport are reflected by the terms inside the square brackets. The first term is the second-order velocity, $\left(K_{d} \zeta_{0} / R\right) u^{\prime}$, which in general is smaller in magnitude than $u^{\prime}$. Hence the chemical advection is also effectively retarded by sedimentation, but to a lesser extent. The second term can change in sign depending on the sediment concentration gradient. Practically the sediment and the chemical concentration gradients would have the same sign in most of their distributions. Therefore, the advective flux of the chemical is reduced or increased on the part where the concentration gradient is positive or negative. This in effect enlarges the dispersion of the chemical species.

In short, the effective velocity and dispersion coefficient for the chemical transport depend on the local sediment concentration, and therefore change with space and time. Equation (65) is an original result that has not been developed previously by heuristic arguments.

The association of the chemical transport with the sediment transport ceases only when the sediment and the chemical clouds are completely separated (i.e., the sediment particles are thoroughly cleansed), if possible. By then the retardation factor becomes unity and the chemical transport equation reduces to

$$
\frac{\partial C_{0}}{\partial t}+\bar{u} \frac{\partial C_{0}}{\partial x}=\varepsilon\left(\bar{E}_{x}+D\right) \frac{\partial^{2} C_{0}}{\partial x^{2}} .
$$

For simplicity, we shall in the following example drop the leading order subscript. Also the eddy diffusivity $\bar{E}_{x}$ will be ignored as it is much smaller than the dispersion coefficient $D$.

\section{A PULSE INPUT OF CONTAMINATED SEDIMENT}

For illustration, we consider an example in which contaminated particles are discharged to a stream in a pulse input (i.e., an instantaneous plane source, uniformly distributed over the flow cross section). The sediment concentration can be described by a Gaussian distribution in the moving frame $\xi=x-\left(\bar{u}+u^{\prime}\right) t$ :

$$
\zeta(\xi, t)=\frac{m}{\sqrt{4 \pi D t}} \exp \left(-\frac{\xi^{2}}{4 D t}\right),
$$

where $m$ is the mass of sediment per cross-sectional area of stream. Transforming from $(x, t)$ to $(\xi, t)$, and substituting (51) for $u^{\prime}$, the chemical transport equation becomes

$$
R \frac{\partial C}{\partial t}+\left[\frac{\pi^{2} w_{f}}{6 \kappa^{2}}-K_{d} D \frac{\partial \zeta}{\partial \xi}\right] \frac{\partial C}{\partial \xi}=\frac{\partial}{\partial \xi}\left(R D \frac{\partial C}{\partial \xi}\right) .
$$

Note that because of the apparent advection (inside the square brackets), which is of $O(\varepsilon)$, the center of mass of the chemical will be gradually drifting away from that of the sediment.

Let us also assume that before dumping the particles are completely dry and a contaminant is uniformly sorbed onto the solid with a sorbate concentration $C_{\text {so }}$. Immediately after discharge into the stream, dissolution happens instantaneously and the aqueous phase distribution can be found from (4):

$$
C=\frac{C_{\mathrm{so}} \zeta}{1+K_{d} \zeta} \quad \text { as } \quad t \rightarrow 0^{+} .
$$

Note that at all times the conservation of mass requires that

$$
\int_{-\infty}^{\infty} \zeta d \xi=m, \quad \int_{-\infty}^{\infty} C_{\mathrm{tot}} d \xi=C_{\mathrm{so}} m \quad \text { for } t>0,
$$


where $C_{\mathrm{tot}}=\left(1+K_{d} \zeta\right) C=R C$ is the total mass of chemical per bulk volume.

Let us now introduce the following normalized quantities (distinguished by a caret):

$$
\begin{aligned}
& \xi=L \hat{\xi}, \quad t=\left(L^{2} / D\right) \hat{t}, \quad \zeta=(m / L) \hat{\zeta}, \\
& \left(C, C_{\mathrm{tot}}\right)=\left(C_{\mathrm{so}} m / L\right)\left(\hat{C}, \hat{C}_{\mathrm{tot}}\right),
\end{aligned}
$$

where $L$ is a longitudinal length scale, which according to (10) and (11) is of the order $(D / E)^{3 / 2} h$. Also note that the slow time scale $L^{2} / D$ is used in the normalization, since this is the time scale for all processes as observed from the moving coordinate $\xi$. In terms of the normalized quantities, the equations for the sediment spreading and the chemical transport can be written as

$$
\begin{aligned}
& \hat{\zeta}=\frac{1}{\sqrt{4 \pi \hat{t}}} \exp \left(-\frac{\hat{\xi}^{2}}{4 \hat{t}}\right), \\
& R \frac{\partial \hat{C}}{\partial \hat{t}}+\left(\operatorname{Pe}_{f}-\alpha \frac{\partial \hat{\zeta}}{\partial \hat{\xi}}\right) \frac{\partial \hat{C}}{\partial \hat{\xi}}=\frac{\partial}{\partial \hat{\xi}}\left(R \frac{\partial \hat{C}}{\partial \hat{\xi}}\right),
\end{aligned}
$$

and

$$
R=1+\alpha \hat{\zeta},
$$

where

$$
\begin{gathered}
\alpha=K_{d} m / L, \\
\mathrm{Pe}_{f}=\frac{\pi^{2} w_{f} L}{6 \kappa^{2} D} .
\end{gathered}
$$

The normalized initial condition (70) is

$$
\hat{C}=\frac{\hat{\zeta}}{1+\alpha \hat{\zeta}} \quad \text { as } \hat{t} \rightarrow 0^{+} .
$$

Also, the integrals in (71) now become

$$
\int_{-\infty}^{\infty} \hat{\zeta} d \hat{\xi}=1, \quad \int_{-\infty}^{\infty} R \hat{C} d \hat{\xi}=1 \quad \text { for } \hat{t}>0
$$

Clearly the chemical transport is controlled by two dimensionless parameters: $\alpha$ and $\mathrm{Pe}_{f}$. The parameter $\alpha$ is the bulk solid-water distribution ratio of the chemical, which has been assumed to be of order unity when (16) is discussed. The other parameter $\mathrm{Pe}_{f}$ indicates the importance of the fall velocity relative to the longitudinal dispersion. Again from previous discussion, this parameter can be of order unity.

Equation (74) is solved numerically using the Crank Nicolson finite-difference method. The conservation of mass equations (79) has been checked by numerical integrations. Small enough spatial discretization and time step are used so that the maximum error is below $1 \%$. To see the various effects, results have been generated for three cases of parameters: (I) $\mathrm{Pe}_{f}=1.0, \alpha=0.1$; (II) $\mathrm{Pe}_{f}=1.0, \alpha=5.0$; (III) $\mathrm{Pe}_{f}=0.1, \alpha=5.0$. From case I, we expect to see the effects of a large fall velocity but a relatively small bulk solid-water distribution ratio. The effects of increasing the bulk solidwater distribution ratio and decreasing the fall velocity are to be seen in cases II and III, respectively. The concentration

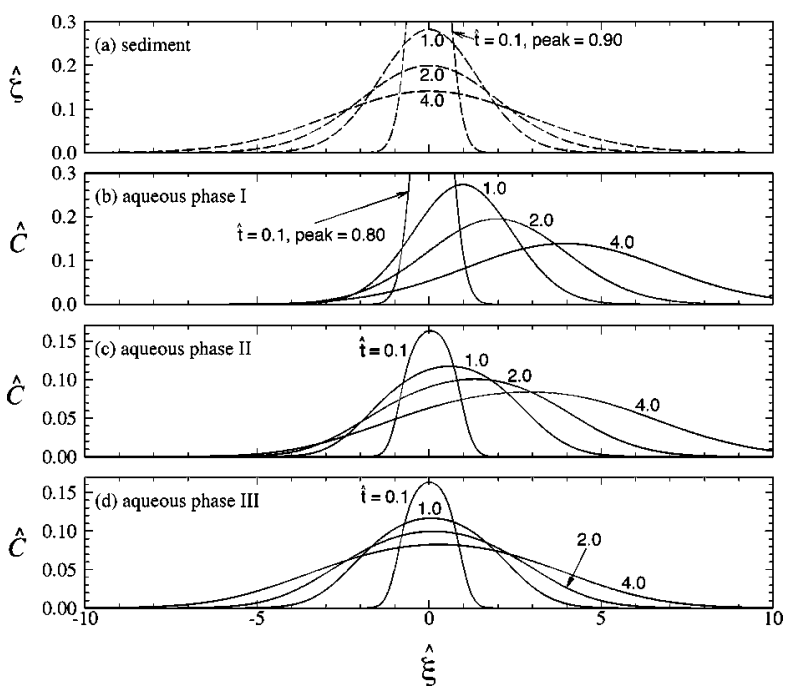

FIG. 1. Concentration distributions as a function of time: (a) Gaussian distributions for sediment; (b) aqueous phase concentration for case I ( $\left.\mathrm{Pe}_{f}=1.0, \alpha=0.1\right)$; (c) aqueous phase concentration for case II ( $\mathrm{Pe}_{f}=1.0$, $\alpha=5.0)$; (d) aqueous phase concentration for case III $\left(\mathrm{Pe}_{f}=0.1, \alpha=5.0\right)$.

distributions as a function of time are shown in Fig. 1, where in (a) are the normal distributions of the sediment concentration $\hat{\zeta}$.

For a large fall velocity and a small $\alpha$ as in case I, the aqueous phase cloud appears to move readily ahead of the sediment cloud. This is reasonable since the apparent velocity of the aqueous phase cloud is proportional to $w_{f} / R$, which is the largest in case I. A small $\alpha$ can mean a higher chemical solubility in water. Therefore as the chemical readily dissolves in water, the sediment will become largely clean when the two clouds are separating from each other.

When the solid-water distribution ratio is larger, so is the retardation. Therefore in case II the aqueous phase cloud drifts less rapidly apart from the sediment cloud. The dispersion is however more extensive, as evident from the more rounded crests of the distributions than in case I. Because of the spatial dependence of dispersion, the spreading is asymmetrical about its peak. When the fall velocity is reduced as in case III, the aqueous phase cloud is essentially moving at the same speed as the sediment cloud. Also the dispersion is not as large as in case II.

We confirm the above-mentioned observations by also examining the location of the center of mass $\hat{\xi}_{c}$ and the variance $\sigma^{2}$ of the distributions. These two quantities, in dimensionless form, can be calculated from

$$
\hat{\xi}_{c}=\frac{\mu_{1}}{\mu_{0}}, \quad \sigma^{2}=\frac{\mu_{2}}{\mu_{0}}-\hat{\xi}_{c}^{2},
$$

where $\mu_{n}$ is the $n$th moment defined by

$$
\mu_{n}=\int_{-\infty}^{\infty} \hat{\xi}^{n} \hat{C} d \hat{\xi}
$$

By a normal distribution, the center of mass of the sediment cloud is always at $\hat{\xi}=0$, and the dimensionless variance is $\sigma^{2}($ sediment $)=2 \hat{t}$. 


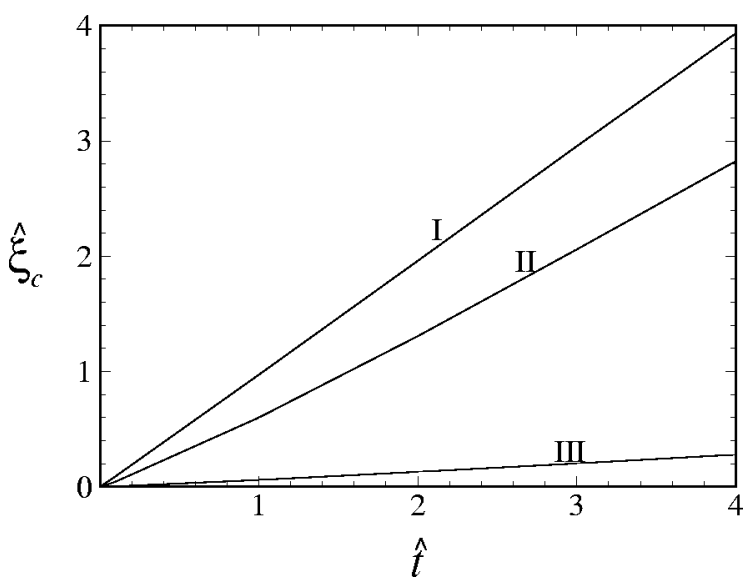

FIG. 2. The locations of the center of mass of the aqueous phase cloud as a function of time for the three cases.

For cases I-III, Figs. 2 and 3 show $\hat{\xi}_{c}$ and $\sigma^{2}$, respectively, as a function of time. The results are essentially consistent with the above-mentioned observations. The separation of the two clouds is the fastest in case I with a large fall velocity and a small solid-water distribution ratio, and the slowest in case III with a small fall velocity and a large solid-water distribution ratio. The dispersion of the dissolved phase cloud is however the largest in case II with a large fall velocity and a large solid-water distribution ratio. The dispersion in case I is close to the dispersion of the sediment cloud.

In the long run the effect of the sorptive exchange will diminish when the two clouds are mostly nonoverlapping. By then the separation speed of the two clouds will only depend on the fall velocity. Also, the enhancement factor of the dispersion coefficient will drop to unity. By virtue of this, we anticipate that curves I and II in Fig. 2 will become straight and parallel to each other at large times. Also at large times the three curves I, II, and III in Fig. 3 will be parallel to the one for sediment with a slope of 2 .

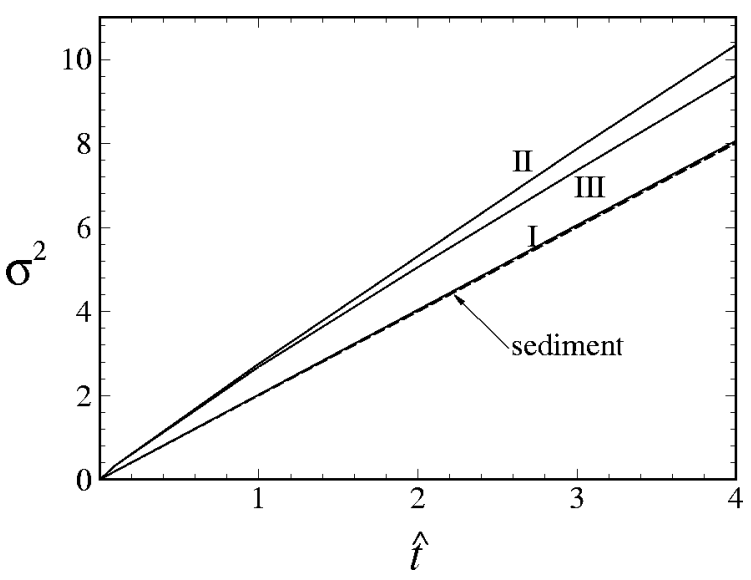

FIG. 3. The variances of the aqueous phase spreading as a function of time for the three cases.

\section{CONCLUDING REMARKS}

Using an asymptotic method of averaging based on the homogenization theory, we have systematically deduced the advective-dispersive transport equations (45) and (65) for suspended sediment and a chemical in a two-dimensional, steady and uniform open-channel flow. The mass transport of the chemical is shown to be influenced by the sediment transport because of sorptive exchange. At the leading order, both substances are advected at the mean stream velocity. At the higher order, the sediment advection is hindered by a speed of approximately ten times the fall velocity, which causes the dissolved phase cloud to gradually move ahead of the sediment cloud. The retardation factor, effective advection velocity, and dispersion coefficient for the chemical transport are functions of space and time as they depend on the local sediment concentration. The results are original and should be of great potential value in water quality modeling.

With a numerical example, we have confirmed the effects of the fall velocity and the bulk solid-water distribution ratio. For larger particulates and a higher fraction of chemical in water, the two clouds will separate from each other more quickly. On the other hand the dispersion of the dissolved chemical is larger for larger particulates and a higher fraction of chemical on solid; the enhancement of the dispersion by the sorption effect is clearly demonstrated.

In this study, we have only considered rather fine particles so that a small fall velocity $w_{f}=O\left(\varepsilon^{3 / 2}\right) \bar{u}$ can be assumed. This leads to a uniform sediment concentration profile at the leading order, and a retardation to advection at the second order. The orders of the results however may change for particles that are an order of magnitude larger. For coarser particles such that, say $w_{f}=O\left(\varepsilon^{1 / 2}\right) \bar{u}$, the sediments will tend to be more concentrated near the channel bottom and consequently the fall velocity can have an $O(1)$ effect on the advection. In addition, the kinetics of sorptive exchange will be more significant for larger particles. These effects may lead to interesting results and will be examined in a future study.

Also we have focused only on the effects of phase exchange between dissolved and particulate forms of a chemical on its transport. For future extensions, it will be desirable if other effects such as volatilization, diffusive exchange with bed sediment, biodegradation, and photolysis can also be included. More challenging work is to consider as well the kinetics of these processes, bedform of the channel, and deposition and resuspension of the sediment particles.

\section{ACKNOWLEDGMENTS}

This research was initiated with support by a CRCG Research Grant awarded by the University of Hong Kong (Grant No. 10202592/31084/14500/323/01), and completed with support by a grant from the Research Grants Council of the Hong Kong Special Administrative Region, China (Project No. HKU 7117/99E). 


\section{APPENDIX: SOLUTIONS OF THE FUNCTIONS $N$ AND $M$, AND EXPRESSIONS FOR $D$ AND $u^{\prime}$}

On integrating (35) and (37) twice with respect to $z$ and using the respective boundary conditions (36) and (38), we get

$$
\begin{aligned}
& N(z)=N(0)+\int_{0}^{z} \frac{d z^{\prime}}{E_{z}\left(z^{\prime}\right)} \int_{0}^{z^{\prime}}\left[u\left(z^{\prime \prime}\right)-\bar{u}\left(z^{\prime \prime}\right)\right] d z^{\prime \prime}, \\
& M(z)=M(0)-w_{f} \int_{0}^{z} \frac{d z^{\prime}}{E_{z}\left(z^{\prime}\right)} .
\end{aligned}
$$

Uniqueness conditions are required in order to determine $N(0)$ and $M(0)$. These constants nonetheless have no influence when determining $D$ and $u^{\prime}$ :

$$
\begin{aligned}
D & =\bar{N} \bar{u}-\overline{N u}=\overline{(N-N(0))(\bar{u}-u)} \\
& =-\frac{1}{h} \int_{0}^{h} d z(u-\bar{u}) \int_{0}^{z} \frac{z z^{\prime}}{E_{z}} \int_{0}^{z^{\prime}}(u-\bar{u}) d z^{\prime \prime}, \\
u^{\prime} & =\overline{M u}-\bar{M} \bar{u}=\overline{(M-M(0))(u-\bar{u})} \\
& =-\frac{w_{f}}{h} \int_{0}^{h} d z(u-\bar{u}) \int_{0}^{z} \frac{d z^{\prime}}{E_{z}} .
\end{aligned}
$$

Using integration by parts, we may change the above integrals to:

$$
\begin{aligned}
& D=\frac{1}{h} \int_{0}^{h} \frac{d z}{E_{z}}\left[\int_{z}^{h}(u-\bar{u}) d z^{\prime}\right]^{2}, \\
& u^{\prime}=-\frac{w_{f}}{h} \int_{0}^{h} \frac{d z}{E_{z}} \int_{z}^{h}(u-\bar{u}) d z^{\prime} .
\end{aligned}
$$

For turbulent open-channel flows, we may use the following velocity defect law, which is valid for both smooth and rough bottoms,

$$
u-\bar{u}=\frac{u_{*}}{\kappa}\left(\ln \frac{z}{h}+1\right),
$$

where $\kappa=0.41$ is von Karman's constant. Also by Reynolds analogy, the eddy diffusivity is equal to the eddy viscosity given by the parabolic distribution

$$
E_{z}(z)=u_{*} \kappa h \frac{z}{h}\left(1-\frac{z}{h}\right) .
$$

Substituting these relations, the integrations can now be worked out readily:

$$
\begin{aligned}
D & =\frac{u_{*} h}{\kappa^{3}} \int_{0}^{1} \frac{\chi}{1-\chi}(\ln \chi)^{2} d \chi \\
& =\frac{u_{*} h}{\kappa^{3}} \sum_{n=1}^{\infty} \int_{0}^{1} \chi^{n}(\ln \chi)^{2} d \chi \\
& =\frac{2 u_{*} h}{\kappa^{3}} \sum_{n=2}^{\infty} \frac{1}{n^{3}}=5.86 u_{*} h,
\end{aligned}
$$

where the value of the series was given by Elder, ${ }^{3}$ and

$$
u^{\prime}=\frac{w_{f}}{\kappa^{2}} \int_{0}^{1} \frac{\ln \chi}{1-\chi} d \chi=-\frac{\pi^{2}}{6 \kappa^{2}} w_{f}=-9.79 w_{f} .
$$

${ }^{1}$ G. I. Taylor, "Dispersion of soluble matter in solvent flowing slowly through a tube," Proc. R. Soc. London, Ser. A 219, 186 (1953).

${ }^{2}$ G. I. Taylor, "The dispersion of matter in turbulent flow through a pipe," Proc. R. Soc. London, Ser. A 223, 446 (1954).

${ }^{3}$ J. W. Elder, "The dispersion of marked fluid in turbulent shear flow," J. Fluid Mech. 5, 544 (1959).

${ }^{4}$ J. L. Schnoor, Environmental Modeling (Wiley, New York, 1996).

${ }^{5}$ R. Aris, "On the dispersion of a solute in a fluid flowing through a tube," Proc. R. Soc. London, Ser. A 235, 67 (1956).

${ }^{6} \mathrm{E}$. Sanchez-Palencia, "Comportement local et macroscopique dùn type de milieu physiques héterogénes,’’ Int. J. Eng. Sci. 12, 331 (1974).

${ }^{7}$ A. Bensoussan, J. L. Lions, and G. Papanicolaou, Asymptotic Analysis for Periodic Structures (North-Holland, Amsterdam, 1978).

${ }^{8}$ C. C. Mei, J. L. Auriault, and C. O. Ng, "Some Applications of the homogenization theory," Adv. Appl. Mech. 32, 277 (1996).

${ }^{9} \mathrm{R}$. Mauri, "Dispersion, convection, and reaction in porous media," Phys. Fluids A 3, 743 (1991).

${ }^{10} \mathrm{C}$. C. Mei, "Method of homogenization applied to dispersion in porous media," Transp. Porous Media 9, 261 (1992).

${ }^{11}$ J. L. Auriault and P. M. Adler, "Taylor dispersion in porous media: Analysis by multiple scale expansions," Adv. Water Resour. 18, 217 (1995).

${ }^{12}$ C. K. Lee, C. C. Sun, and C. C. Mei, "Computation of permeability and dispersivities of solute or heat in periodic porous media," Int. J. Heat Mass Transf. 39, 661 (1996).

${ }^{13}$ U. Hornung, Homogenization and Porous Media (Springer, New York, 1997).

${ }^{14}$ C. O. Ng, "Macroscopic equations for vapor transport in a multi-layered unsaturated zone," Adv. Water Resour. 22, 611 (1999).

${ }^{15}$ C. C. Mei and C. Chian, "Dispersion of small suspended particles in a wave boundary layer," J. Phys. Oceanogr. 24, 2479 (1994).

${ }^{16}$ C. C. Mei, S. J. Fan, and K. R. Jin, "Resuspension and transport of fine sediments by waves," J. Geophys. Res. 102, 15807 (1997).

${ }^{17}$ W. W. Sayre, Dispersion of Mass in Open-Channel Flow (Colorado State University Press, Colorado, 1975).

${ }^{18}$ L. C. van Rijn, “Sediment transport. II. Suspended load transport,' Journal of Hydraulic Engineering 110, 1613 (1984).

${ }^{19}$ P. D. Komar and C. E. Reimers, "Grain shape effects on settling rates," J. Geol. 86, 193 (1978).

${ }^{20}$ R. P. Schwarzenbach, P. M. Gschwend, and D. M. Imboden, Environmental Organic Chemistry (Wiley, New York, 1993)

${ }^{21}$ J. C. Means, S. G. Wood, J. J. Hassett, and W. L. Banwart, "Sorption of polynuclear aromatic hydrocarbons by sediments and soils," Environ. Sci. Technol. 14, 1524 (1980).

${ }^{22}$ S. C. Wu and P. M. Gschwend, "Sorption kinetics of hydrophobic organic compounds to natural sediments and soils," Environ. Sci. Technol. 20, 717 (1986).

${ }^{23}$ V. A. Vanoni, Sedimentation Engineering (ASCE Task Comm., New York, 1975) 